\title{
Speed selection for traveling-wave solutions to the diffusion-reaction equation with cubic reaction term and Burgers nonlinear convection
}

\author{
V. A. Sabelnikov \\ ONERA - The French Aerospace Laboratory, F-91761 Palaiseau, France \\ A. N. Lipatnikov \\ Department of Applied Mechanics, Chalmers University of Technology, Gothenburg, 412 96, Sweden
}

(Received 4 June 2014; published 9 September 2014)

\begin{abstract}
The problem of traveling wave (TW) speed selection for solutions to a generalized Murray-Burgers-KPP-Fisher parabolic equation with a strictly positive cubic reaction term is considered theoretically and the initial boundary value problem is numerically solved in order to support obtained analytical results. Depending on the magnitude of a parameter inherent in the reaction term (i) the term is either a concave function or a function with the inflection point and (ii) transition from pulled to pushed TW solution occurs due to interplay of two nonlinear terms; the reaction term and the Burgers convection term. Explicit pushed TW solutions are derived. It is shown that physically observable TW solutions, i.e., solutions obtained by solving the initial boundary value problem with a sufficiently steep initial condition, can be determined by seeking the TW solution characterized by the maximum decay rate at its leading edge. In the Appendix, the developed approach is applied to a non-linear diffusion-reaction equation that is widely used to model premixed turbulent combustion.
\end{abstract}

DOI: 10.1103/PhysRevE.90.033004

PACS number(s): 47.54.-r, 47.10.-g, 82.40.Ck, 87.10.-e

\section{INTRODUCTION}

Propagation of a wave in a nonequilibrium medium is a widespread phenomenon relevant to many branches of science, such as biology [1-4], economics [5], combustion [6,7], chemistry [8,9], physics [10], etc. To model the phenomenon, the following nonlinear diffusion-reaction equation:

$$
\frac{\partial u}{\partial t}=D \frac{\partial^{2} u}{\partial x^{2}}+Q(u)
$$

is widely used, with the first and second terms on the right hand side (RHS) describing the spread of the wave due to diffusion of a normalized density of a substance $u$ with a constant coefficient $D$ and nonlinear reaction rate $Q(u)$, respectively. Here, $t \geqslant 0$ is time, independent variable $x$ is unbounded, i.e., $-\infty<x<\infty$, the function $u(x, t)$ characterizes the state of the medium, with $u=0$ and $u=1$ being homogeneous unstable and stable states, respectively, i.e.,

$$
Q(0)=Q(1)=0, \quad Q(0<u<1)>0,
$$

and, therefore, the derivatives $Q^{\prime}(0)>0, Q^{\prime}(1)<0$. It is worth noting that other reaction terms, which were not strictly positive, were also considered in the literature, e.g., see $[5,6,11,12]$ and references quoted therein. The present study is restricted to strictly positive reaction terms.

For dimensional reasons, the non-linear reaction (source) term $Q(u)$ can be written as follows:

$$
Q(u)=\frac{\omega(u)}{\tau_{f}},
$$

where $\tau_{f}$ is a constant time scale. Subsequently, Eq. (1) reads

$$
\frac{\partial u}{\partial \tau}=\frac{\partial^{2} u}{\partial \xi^{2}}+\omega(u),
$$

where $\tau=t / \tau_{f}$ and $\xi=x / \sqrt{D \tau_{f}}$ are the normalized time and spatial coordinate, respectively.
Partial differential Eq. (4) admits traveling-wave (TW) solutions of permanent monotonous shape $u(\xi, \tau)=U(\zeta)$, where $\zeta=\xi-\Lambda \tau$ is the wave variable. Such a TW body propagates into an unstable state with a constant speed $\Lambda>0$ (for brevity, we consider TWs that propagate from left to right). Substitution of the TW solution to Eq. (4) results in the following ordinary differential equation:

$$
-\Lambda \frac{d U}{d \zeta}=\frac{d^{2} u}{d \zeta^{2}}+\omega(u)
$$

which is written in the coordinate framework attached to the TW. Boundary conditions read

$$
U(-\infty)=1, \quad U(\infty)=0 .
$$

Depending on the shape of the nonlinear reaction term $\omega(U)$, two regimes of TW propagation can be distinguished, i.e., the so-called pulled and pushed TWs, e.g., see a review paper by Ebert and van Saarloos [13]. The name pulled TW comes from the fact that the speed spectrum and, in particular, minimum (slowest) speed can be found using a linear analysis technique in the vicinity of the unstable point, i.e., by linearizing Eq. (5) with respect to $U \ll 1$ at the leading edge of the TW. In other words, such a TW is pulled along its leading edge. To the contrary, the speed of a pushed TW is controlled by the entire wave structure and depends on the shape of the nonlinear reaction term $\omega(U)$ at $0<U<1$.

Research into the pulled TW solutions to Eqs. (5)-(6) was pioneered by Kolmogorov, Petrovsky, and Piskounov [2] who studied the spreading of an advantageous gene in a population by considering the following source term:

$$
\begin{aligned}
& \omega(0)=\omega(1)=0, \quad \omega(0<u<1)>0, \\
& \omega^{\prime}(0<u \leqslant 1)<\omega^{\prime}(0) .
\end{aligned}
$$

The last inequality in Eq. (7) means that the source term $\omega$ has the highest slope at $u=0$. It is assumed further without loss of generality that $\omega^{\prime}(0)=1$. Equation (7) is satisfied for a wide 
class of source terms, e.g., for concave functions, which have negative second derivative $\omega^{\prime \prime}$ in the interval $0<u<1$. A widely used model of such a concave source term is as follows $\omega=u-u^{k}$, where $k>1$. Equations (5)-(7) are commonly called the KPP case of a general diffusion-reaction problem.

Kolmogorov et al. [2] have shown that the nonlinear boundary value problem given by Eqs. (5)-(7) cannot uniquely determine the physically realizable (observable) wave speed. They have established that the boundary value problem has a spectrum $\Lambda \geqslant \Lambda_{\min }$ of the wave speeds, bounded by a minimum (slowest) speed $\Lambda_{\min }=\Lambda_{\mathrm{KPP}}=2 \sqrt{\omega^{\prime}(0)}=2$. As $\zeta \rightarrow \infty$, the TW solutions $U(\zeta)$ decay at exponential rate associated with the $\kappa_{-}$branch of the dispersion relation $\kappa^{2}-\Lambda \kappa+1=0$, which results from the linear analysis [2]. Here, $\kappa_{-}=\left(\Lambda-\sqrt{\Lambda^{2}-4}\right) / 2 \leqslant 1$. The decay rate attains a maximum $\kappa_{\mathrm{KPP}}=1$ for the minimum speed $\Lambda_{\mathrm{KPP}}=2$.

As already mentioned, the spectrum $\Lambda \geqslant \Lambda_{\mathrm{KPP}}=2$ can be found using the linear analysis technique in the vicinity of the unstable point, i.e., by linearizing Eq. (5) with respect to $U \ll 1$ at the leading edge. A natural question arises: what TW is actually observed? To answer this question, i.e., to select the physically realizable (observable) wave speed, Kolmogorov et al. [2] studied the long-time behavior of the solutions to the initial boundary value problem given by Eq. (4) with the nonlinear source term $\omega(u)$ that satisfies Eq. (7). They have shown that for physically natural initial conditions associated with a sufficiently steep initial profile of $u(x, t=0)=u_{0}(x)$, e.g., $u_{0}(x)=1-H(x)=H(-x)$, where $H$ is Heaviside function, the solution to the initial boundary value problem approaches the TW solution characterized by the slowest speed $\Lambda_{\mathrm{KPP}}=2$ and the highest decay rate $\kappa_{\mathrm{KPP}}=1$.

It is worth stressing that a TW is a self-similar intermediate asymptotic of the long-time solution $u(\xi, \tau)=v(\xi-\Lambda \tau, \tau)=$ $v(\zeta, \tau)$ to the initial boundary value problem. Accordingly, in order to investigate the asymptotic behavior of the long-time solution $u(\xi, \tau)$ at the leading edge, two limits of $\xi \rightarrow \infty$ and $\tau \rightarrow \infty$ should be considered. This problem is discussed in detail in a review paper by Ebert and van Saarloos [13]. The authors showed that the two limits do not commute and, consequently, the order of the limits is of importance. The linear analysis of a TW at the leading edge, which yields $U \propto$ $\exp (-\kappa \zeta)$, is valid for $1 \ll \zeta_{t} \equiv \zeta-1.5 \ln \tau \ll \sqrt{\tau}$, while at $\zeta_{t} \gg 2 \sqrt{\tau} \gg 1$, the solution $u(\xi, \tau)$ decays as a Gaussian function $u(\xi, \tau) \propto \exp \left(-\xi^{2} / 4 \tau\right)$, which is steeper than any exponential one. The interested reader is referred to the book by Barenblatt [14] for further discussion of the intermediate asymptotics and examples.

Another regime of TW propagation was studied by Aronson and Weinberger [5] who addressed a more general reaction (source) term

$$
\omega(0)=\omega(1)=0, \quad \omega(0<u<1)>0, \quad \omega^{\prime}(0)>0 .
$$

This constraint allows the reaction term $\omega$ to have an inflection point in the interval $0<u<1$, i.e., the maximum slope of $\omega(u)$ can be reached at $0<u<1$. Aronson and Weinberger [5] have proved that: (i) similarly to the KPP case, the boundary value problem given by Eqs. (5), (6), and (8) has a spectrum $\Lambda \geqslant \Lambda_{\min }$ of TW speeds, bounded by the minimum (slowest) speed, which satisfies the following constraints:

$$
\Lambda_{\mathrm{KPP}} \leqslant \Lambda_{\min } \leqslant 2 \sup _{0<u<1} \sqrt{\frac{\omega(u)}{u}} .
$$

It should be stressed that Eq. (9) gives only bounds for $\Lambda_{\min }$, but there is no explicit expression that relates the slowest speed $\Lambda_{\text {min }}$ to the parameters of the boundary value problem. In the KPP case, the lower and upper bounds coincide and, consequently, $\Lambda_{\min }=\Lambda_{\mathrm{KPP}}$, but this equality can hold even if the lower and upper bounds are different.

Similarly to the KPP case, TW solutions to Eqs. (5), (6), and (8) that have $\Lambda>\Lambda_{\text {min }}$ decay exponentially, with the decay rate belonging to the $\kappa_{-}$branch of the dispersion relation $\kappa^{2}-$ $\Lambda \kappa+1=0$, which results from the linear analysis. Moreover, TW solutions with the slowest speed can be associated with an isolated discrete point $\Lambda=\Lambda_{\text {min }}$ and decay with a rate $\kappa_{+}=\left(\Lambda+\sqrt{\Lambda^{2}-4}\right) / 2$, which belongs to another branch of the dispersion relation [5].

In order to find TW solution that is actually observed, Aronson and Weinberger [5] analyzed the long-time behavior of the solutions to the initial boundary value problem given by Eq. (4) with the nonlinear source term $\omega(u)$ that satisfies Eq. (8). They proved that, for the natural (sufficiently steep) initial profile of $u_{0}(x)$, the solution to the initial boundary value problem can tend to pushed TW with $\Lambda=\Lambda_{\text {min }}$ and decay rate $\kappa_{+}>1$ larger than $\kappa_{\mathrm{KPP}}=1$.

Murray $[3,4]$ was the first who introduced a nonlinear scalar flux $J(u)$ with the derivative $J^{\prime} \neq$ const into the diffusionreaction Eq. (1), i.e., he investigated the following equation:

$$
\frac{\partial u}{\partial t}+\frac{\partial J(u)}{\partial x}=D \frac{\partial^{2} u}{\partial x^{2}}+\frac{\omega(u)}{\tau_{f}},
$$

which is known as quasi-linear convection diffusion-reaction equation and can be rewritten as follows:

$$
\frac{\partial u}{\partial t}+J^{\prime} \frac{\partial u}{\partial x}=D \frac{\partial^{2} u}{\partial x^{2}}+\frac{\omega(u)}{\tau_{f}} .
$$

Introduction of the flux $J(u)$ into Eq. (11) aims at allowing for deterministic motion of substance in (or opposite to) the direction of its gradient, in addition to the Brownian motion modeled by the first term on the RHS. Such deterministic motion is relevant to various phenomena in biology, physiology, chemistry, etc., e.g., see [15-18].

The effect of the nonlinear convection term $J^{\prime} \partial u / \partial x$ is well known in the particular case of $D=\omega=0$. In that case, Eq. (11) is transformed to a quasi-linear hyperbolic equation [19-21]. If the slope $J^{\prime}$ of the nonlinear convection term is positive (negative) and the initial profile $u_{0}(x)$ is a smooth decreasing function, i.e., $d u_{0} / d x<0$, then the profile $u(x, t)$ is compressed (expanded) and a shock (rarefaction) wave is generated.

By analyzing Eq. (10) with $\omega(u)=u(1-u)$ and $J(u)=$ $K u^{2} / 2$ or $J^{\prime}=K u$, where parameter $-\infty<K<\infty$ had dimension of velocity, Murray [3,4] found that depending on the value of $K$, not only pulled, but also pushed TW solutions satisfied Eq. (10). Murray's quasi-linear convection 
diffusion-reaction equation reads

$$
\frac{\partial u}{\partial t}+K u \frac{\partial u}{\partial x}=D \frac{\partial^{2} u}{\partial x^{2}}+\frac{u(1-u)}{\tau_{f}}
$$

or

$$
\frac{\partial u}{\partial \tau}+k u \frac{\partial u}{\partial \xi}=\frac{\partial^{2} u}{\partial \xi^{2}}+u(1-u)
$$

in a dimensionless form, where $k=K / \sqrt{D / \tau_{f}}$. Because the left hand side (LHS) of Eq. (13) is the well-known inviscid Burgers equation [19-21], Eq. (13) can be called the Burgers-KPP-Fisher equation. Accordingly, the parameter $k$ is the normalized intensity of the Burgers convection term.

The boundary value problem for TW solutions to Eq. (13) reads

$$
-\Lambda \frac{d U}{d \zeta}+k U \frac{d U}{d \zeta}=\frac{d^{2} U}{d \zeta^{2}}+U(1-U),
$$

with the boundary conditions being given by Eq. (6). By investigating the boundary value problem, Murray $[3,4]$ has shown that if $k \leqslant k_{c r}=2$, then (i) TW solutions are pulled, (ii) the spectrum $\Lambda \geqslant \Lambda_{\text {min }}$ of TW speeds is bounded by a minimum speed $\Lambda_{\min }=\Lambda_{\mathrm{KPP}}=2$, and (iii) the decay rates of the solutions belong to the $\kappa_{-}$-branch of the dispersion relation $\kappa^{2}-\Lambda \kappa+1=0$, which results from the linear analysis. All these results are similar to the KPP results. However, if $k>k_{c r}$, then (i) the slowest wave speed is given by $\Lambda_{\min }=$ $k / 2+2 / k>\Lambda_{\mathrm{KPP}}=2$, (ii) the TWs with $\Lambda=\Lambda_{\min }$ are pushed, and (iii) have the decay rates $\kappa_{+}=k / 2>\kappa_{\mathrm{KPP}}=1$, which belong to another branch of the dispersion relation. Subsequently, Murray [3,4] numerically solved the initial boundary value problem for Eq. (11) with the Heaviside-like initial profile $u_{0}(x)$ for a few representative values of the parameter $K$ and found that the numerical solutions tended to (i) the pulled TW with $\Lambda=\Lambda_{\min }=\Lambda_{\mathrm{KPP}}=2$ and $\kappa=\kappa_{\mathrm{KPP}}$ when $k \leqslant k_{c r}=2$, but (ii) the pushed TWs with $\Lambda=\Lambda_{\min }=$ $k / 2+2 / k>\Lambda_{\mathrm{KPP}}=2$ and $\kappa=\kappa_{+}=k / 2>\kappa_{\mathrm{KPP}}=1$ when $k>k_{c r}$. Recent numerical simulations by Méndez and Fort [22] validated these findings also.

These results imply that the logistic reaction term $\omega(u)=$ $u(1-u)$ overwhelms the Burgers nonlinear convection term $k u \partial u / \partial \xi$ if $k<k_{c r}$, whereas the Burgers nonlinear convection term overwhelms the logistic reaction term if $k>k_{c r}$.

From the above discussion, it is clear that the boundary value problem for either the diffusion-reaction or quasi-linear convection diffusion-reaction equation does not uniquely determine the TW speed. The speed selection is performed by solving the counterpart initial boundary value problem theoretically and/or numerically. For physically natural initial conditions, e.g., given by Heaviside function, the TW with the slowest speed $\Lambda_{\min }$ and the highest decay rate is commonly selected.

Many efforts were performed to resolve the speed selection problem within the framework of the boundary value problem, as an alternative to the consideration of the initial boundary value problem. Here, we can mention (i) the marginal stability hypothesis [23-25] and closely related steepness criterion [26-28], (ii) construction of explicit solutions [28,29], (iii) the structural stability hypothesis [30], (iv) dynamical system methods [31], and (v) variational methods [32,33]. The interested reader is referred to a review paper by von Saarloos [34] for further discussion of the speed selection problem and examples.

In the present communication, we support the steepness criterion [26-28] by applying it to the problem of speed selection for a generalized Murray-Burgers-KPP-Fisher Eq. (13) with a cubic reaction term which, depending on a parameter, can be either a concave function or a function with the inflection point. In this case, transition from pulled to pushed TWs is controlled by the interplay of two nonlinear terms, i.e., the Burgers convection and reaction terms.

The paper is organized as follows. Section II contains a qualitative discussion of a role played by the Burgers convection term in the problem of front propagation into unstable states. In Sec. III, the boundary value problem for finding TW solutions is stated and the linear analysis is applied to it. Explicit pushed TW solutions to the problem are derived in Sec. IV in order to study the interplay between the nonlinear convection and cubic source terms. In Sec. V, we compare the decay rates of pulled and pushed TW solutions in order to select the physically observable TWs, by associating them with waves that are characterized by the highest decay rate. In Sec. VI, we confirm obtained analytical results by numerically solving the initial boundary value problem for Eq. (13). Conclusions are drawn in Sec. VII. The Appendix addresses an application of the obtained results to the theory of premixed turbulent combustion.

\section{MATHEMATICAL FORMULATION AND GOVERNING EQUATIONS}

Let us consider the initial boundary value problem for the quasi-linear convection diffusion-reaction equation with the Burgers convection term, i.e., Eq. (13), and with a cubic reaction term

$$
\omega=u(1-u)(1+2 \varepsilon u)
$$

where $\varepsilon \geqslant-0.5$ in order for the reaction term to be positive for $0<u<1$. The reaction term is not concave if $\varepsilon>0.5$. Equation (15) subsumes the following particular cases: $\omega=$ $u(1-u)^{2}$ if $\varepsilon=-0.5, \omega=u(1-u)$ if $\varepsilon=0$, or $\omega=u(1-$ $\left.u^{2}\right)$ if $\varepsilon=0.5$.

It is worth noting that a cubic reaction term that is not strictly positive is also used in various applications. For instance, the FitzHugh-Nagumo model of the transmission of nerve impulses [35,36] is based on Eq. (4) with $\omega=$ $u(1-u)(u-\alpha)$, where $0<\alpha<1$. Equation (4) with that reaction term is called the FitzHugh-Nagumo equation. In this case, the reaction term is negative in an interval of $0<u<\alpha$, points $u=0$ and $u=1$ are stable, whereas point $u=\alpha$ is unstable.

Substitution of Eq. (15) into Eq. (13) yields

$$
\frac{\partial u}{\partial \tau}+k u \frac{\partial u}{\partial \xi}=\frac{\partial^{2} u}{\partial \xi^{2}}+u(1-u)(1+2 \varepsilon u) .
$$

Boundary conditions are as follows:

$$
u(-\infty, \tau)=1, \quad u(\infty, \tau)=0 .
$$


Integration of Eq. (16) over interval $\xi_{1}<\xi<\xi_{2}$ results in the following integral form of the scalar conservation law:

$$
\begin{array}{r}
\frac{d}{d \tau} I_{u}\left(\xi_{1}, \xi_{2}, \tau\right)+\frac{k}{2} u^{2}\left(\xi_{2}, \tau\right)-\frac{k}{2} u^{2}\left(\xi_{1}, \tau\right) \\
-\left(\left.\frac{\partial u}{\partial \xi}\right|_{\xi_{2}}-\left.\frac{\partial u}{\partial \xi}\right|_{\xi_{1}}\right)=\int_{\xi_{1}}^{\xi_{2}} \omega(u) d \xi,
\end{array}
$$

where

$$
I_{u}\left(\xi_{1}, \xi_{2}, \tau\right) \equiv \int_{\xi_{1}}^{\xi_{2}} u(\xi, \tau) d \xi
$$

If $\xi_{1}=-\infty$ and $\xi_{2}=\infty$, Eqs. (18) and (19) read

$$
\frac{d}{d \tau} I_{u}(-\infty, \infty, \tau)=\frac{k}{2}+\int_{-\infty}^{\infty} \omega(u) d \xi
$$

and

$$
I_{u}(-\infty, \infty, \tau) \equiv \int_{-\infty}^{\infty} u(\xi, \tau) d \xi,
$$

respectively, provided that $\partial u / \partial \xi$ is assumed to vanish at $\xi=-\infty$ and $\xi=\infty$. The quantity $I_{u}(-\infty, \infty, \tau)$ is the total amount of the substance $u(\xi, \tau)$ in the unbounded domain $-\infty<\xi<\infty$. The time derivative of $I_{u}(-\infty, \infty, \tau)$ is well defined in spite of the fact that the integral on the RHS of Eq. (21) is divergent.

Equation (20) contains two terms on the RHS. The first term $k / 2$ depends explicitly on the intensity of the Burgers convection velocity. If $k>0$, this term coincides with the speed of the shock wave generated in Burgers equation, see Eq. (24) below. The second term $\int_{-\infty}^{\infty} \omega(u) d \xi$ is the spatial integral of the reaction term. It depends on the Burgers convection term implicitly, because the latter term affects the profile $u(\xi, \tau)$ by compressing (expanding) it if $k>0(k<0)$. The term $k / 2$ on the RHS of Eq. (20) is equal to the net flux into the region through the left infinity $\xi=-\infty$ (at the right infinity $\xi=\infty$, this flux vanishes due to the boundary condition). Equation (20) shows the difference between explicit and implicit impacts of the Burgers convection term on the time evolution of the global quantity $I_{u}(-\infty, \infty, \tau)$. This twofold effect of the Burgers convection term holds also for the speeds of TW solutions, as will be discussed later.

To further clarify a role played by the Burgers convection term, let us consider abridged Eq. (16), i.e., its LHS

$$
\frac{\partial u}{\partial \tau}+k u \frac{\partial u}{\partial \xi}=0
$$

which is the inviscid Burgers equation [19-21]. In the case of a smooth initial condition, the solution to this equation supplemented with the boundary conditions given by Eq. (17) develops to a shock or rarefaction wave [19-21]. In the particular case of

$$
u(\xi, 0)=u_{0}(\xi)=1-H(\xi)=H(-\xi),
$$

the problem has two types of solutions. If $k>0$, the solution is a singular TW which is nothing but a shock wave propagating at speed $k / 2$, i.e.,

$$
u(\zeta, \theta)=1-H(\zeta)=H(-\zeta), \quad \zeta=\xi-\frac{k}{2} \tau
$$

If $k<0$, the solution is a centered rarefaction wave (not a TW) with the appearance of all intermediate values of $0<u<1$, i.e.,

$$
u(\zeta, \theta)=\left\{\begin{array}{lll}
1 & \text { if } \quad \xi<-|k| / 2 \tau \\
-\xi /(|k| \tau) & \text { if } \quad-|k| / 2 \tau \leqslant \xi \leqslant 0 \\
0 & \text { if } \quad \xi>0
\end{array}\right.
$$

and Eq. (20) reads

$$
\frac{d}{d \tau} I_{u}(-\infty, \infty, \tau)=\frac{k}{2} .
$$

Therefore, the quantity $I_{u}(-\infty, \infty, \tau)$ is not conserved for the inviscid Burgers equation, but increases (decreases) for positive (negative) $k$ due to the supply (withdrawal) of the substance $u$ with the flux $k / 2$ at $\xi=-\infty$.

Solutions to the viscous Burgers equation, i.e., Eq. (13) without the source term on the RHS, supplemented with the initial conditions given by Eq. (23) resemble qualitatively the solutions to the inviscid Burgers equation, given by Eq. (24) or (25), with the shock or rarefaction wave being spread by the diffusion term. The speed of such a spread shock or rarefaction wave is equal to $k / 2$, i.e., it is not affected by the diffusion term.

\section{BOUNDARY VALUE PROBLEM. LINEAR ANALYSIS}

Substitution of a TW solution $u(\xi, \tau)=U(\zeta)$, where $\zeta=$ $\xi-\Lambda \tau$ into the partially differential Eq. (16) yields the following second-order ordinary differential equation:

$$
-\Lambda \frac{d U}{d \zeta}+k U \frac{d U}{d \zeta}=\frac{d^{2} U}{d \zeta^{2}}+U(1-U)(1+2 \varepsilon U)
$$

which jointly with the boundary conditions given by Eq. (6) constitute a boundary value problem.

Integration of Eq. (27) from $\zeta=-\infty$ to $\zeta=\infty$ results in the following equation:

$$
\Lambda=\frac{k}{2}+\int_{-\infty}^{\infty} \omega(U) d \zeta
$$

for the TW speed $\Lambda$ provided that Eq. (6) holds. Thus, as noted above, the TW speed is the sum of two terms, i.e., (i) the explicit contribution $k / 2$ from the Burgers convection term and (ii) spatially integrated reaction term $\int_{-\infty}^{\infty} \omega(U) d \zeta$.

The second-order boundary value problem given by Eqs. (6) and (27) can be reduced to a first-order boundary value problem

$$
-\Lambda P+k U P=P^{\prime} P+U(1-U)(1+2 \varepsilon U),
$$

$$
P(0)=P(1)=0,
$$

which is stated in the phase space $(U, P)$, where $P=d U / d \zeta$ and $P^{\prime}=d P / d U$.

Either the boundary value problem given by Eqs. (6) and (27) or the boundary value problem given by Eqs. (29) and (30) is an eigenvalue problem. We have to find such eigenvalues $\Lambda$ that the obtained trajectory $P(U)$ connects two singular points $(1,0)$ and $(0,0)$ in the phase space $[2,5]$. The complete solution to the boundary value problem requires considering the global behavior of trajectories $P(U)$ in the phase space. However, the necessary conditions for the existence of such trajectories can be found by applying linear analysis at the leading edge. 
To do so, let us linearize Eq. (29) at the leading edge, i.e., in the vicinity of the unstable equilibrium point $U=0[2,5]$. Substitution of

$$
P=-\kappa U, \quad \kappa>0, \quad U \propto \exp (-\kappa \xi), \quad \xi \rightarrow \infty
$$

into Eq. (29) yields the following dispersion relation:

$$
\kappa^{2}-\Lambda \kappa+1=0,
$$

which links the TW speed $\Lambda$ and the decay rate $\kappa$ of the profile $U(\zeta)$ at the leading edge. This dispersion relation has two branches

$$
\begin{gathered}
\kappa_{+}=\frac{\Lambda}{2}+\sqrt{\frac{\Lambda^{2}}{4}-1}, \\
\kappa_{-}=\frac{\Lambda}{2}-\sqrt{\frac{\Lambda^{2}}{4}-1}
\end{gathered}
$$

with $\kappa_{+} \kappa_{-}=1$. Because $U(\zeta)$ is a monotonous positive function, both $\kappa_{+}$and $\kappa_{-}$should be real. Because the dispersion relation given by Eq. (32) depends only on the linear terms in Eq. (29), neither the Burgers convection term, which is of the second order with respect to $U$, nor the second- and third-order contributions to the reaction term play a role, and Eq. (32) coincides with the dispersion relation of the KPP problem $(k=\varepsilon=0)$. Therefore, the linear analysis does not allow us to gain an insight into the influence of these terms on the TW solutions and their speeds. We can only arrive at the well-known KPP result [2], i.e.,

$$
\Lambda_{\min } \geqslant \Lambda_{\mathrm{KPP}}=2 .
$$

\section{EXACT SOLUTION}

Let us find explicit pushed TW solutions to the boundary value problem given by Eqs. (29) and (30) such that the wave speed and decay rate are higher than $\Lambda_{\mathrm{KPP}}$ and $\kappa_{\mathrm{KPP}}$, respectively. We seek a solution $P(U)$ in a form of a quadratic function

$$
\begin{aligned}
P & =-\kappa_{+} U(1-U), \quad P^{\prime}=-\kappa_{+}(1-2 U), \\
U & =\frac{1}{1+\exp \left(\kappa_{+} \xi\right)},
\end{aligned}
$$

where $\kappa_{+} \geqslant 1$ satisfies Eq. (33). Substitution of Eq. (36) into Eq. (29) yields the following relation:

$$
-\Lambda+\kappa_{+}+\frac{1}{\kappa_{+}}+\left(k-2 \kappa_{+}+\frac{2 \varepsilon}{\kappa_{+}}\right) U=0 .
$$

In order for Eq. (37) to be identically satisfied for all $0<$ $U<1$, the following two equations:

$$
-\Lambda+\kappa_{+}+\frac{1}{\kappa_{+}}=0
$$

and

$$
k-2 \kappa_{+}+\frac{2 \varepsilon}{\kappa_{+}}=0
$$

should hold. Note that Eq. (38) is identical to the dispersion relation given by Eq. (32) with $\kappa=\kappa_{+}$. This result is not surprising, because Eq. (32) is always valid at $U \ll 1$.
Equation (39) reads

$$
\kappa_{+}^{2}-\frac{k}{2} \kappa_{+}-\varepsilon=0
$$

Because physically admissible roots of the quadratic Eq. (40) should be positive, we have

$$
\kappa_{+}=\frac{k}{4}+\sqrt{\frac{k^{2}}{16}+\varepsilon}
$$

Because the decay rate $\kappa_{+}$should be real, the TW solutions given by Eqs. (36) and (41) exist only if

$$
\varepsilon>0 \text { or } k>4 \sqrt{|\varepsilon|} \text {. }
$$

If $-1 / 2 \leqslant \varepsilon<0$, then the exact TW solution does not exist in the range of $k<\sqrt{4|\varepsilon|}$.

Equations (38) and (41) result straightforwardly in

$$
\Lambda=\kappa_{+}+\frac{1}{\kappa_{+}} \geqslant 2 \text {. }
$$

In the case of the logistic growth rate, i.e., $\varepsilon=0$, Eqs. (41) and (43) reduce to the well-known exact solution by Murray [3,4],

$$
\kappa_{+}=\frac{k}{2}, \quad \Lambda=\frac{k}{2}+\frac{2}{k} \geqslant 2, \quad k>0 .
$$

In the case of vanishing nonlinear convection term, i.e., $k=$ 0 , Eqs. (41) and (43) reduce to the exact solution found by Hadeler and Rothe [37],

$$
\kappa_{+}=\sqrt{\varepsilon}, \quad \Lambda=\sqrt{\varepsilon}+\frac{1}{\sqrt{\varepsilon}} \geqslant 2, \quad \varepsilon>0 .
$$

\section{SELECTION OF TW SOLUTION}

In line with the steepness criterion [26-28], a physically realizable TW solution to the initial boundary value problem is the TW solution with the maximum decay rate $\kappa$. Accordingly, the inequality $\kappa_{+}>\kappa_{\mathrm{KPP}}$ should be satisfied in order for the explicit pushed TW solution to be physically relevant, where $\kappa_{\mathrm{KPP}}=1$ results from the linear analysis. If $\kappa_{+}<\kappa_{\mathrm{KPP}}$, then, the pulled solution with $\kappa=\kappa_{\mathrm{KPP}}$ is physically realizable. Thus, combining the KPP result for pulled TWs with the exact solution for the pushed TWs, we obtain the following propagation speeds:

$$
\Lambda= \begin{cases}\Lambda_{\mathrm{KPP}}=2 & \text { if } \quad-\infty<k<k_{c r} \\ k / 2+(1+\varepsilon) / \kappa_{+} & \text {if } \quad k_{c r}<k<\infty\end{cases}
$$

and decay rates

$\kappa= \begin{cases}\kappa_{\mathrm{KPP}}=1 & \text { if } \quad-\infty<k<k_{c r} \\ \kappa_{+}=k / 4+\sqrt{k^{2} / 16+\varepsilon} & \text { if } \quad k_{c r}<k<\infty\end{cases}$

of the physically relevant TW solutions, where

$$
k_{c r}=2(1-\varepsilon)
$$

is the critical intensity of the nonlinear Burgers convection term at which the transition from pulled to pushed TW solutions occurs. It is worth remembering that $\varepsilon \geqslant-1 / 2$. Note that Eq. (48) is consistent with the constraints given by Eq. (42). Indeed, if $-1 / 2 \leqslant \varepsilon<0$, then

$$
2(1-\varepsilon)-4 \sqrt{|\varepsilon|}=2+2|\varepsilon|-4 \sqrt{|\varepsilon|}=2(1-\sqrt{|\varepsilon|})^{2}>0 .
$$


Equation (47) shows that if $k>k_{c r}$, then, the decay rate $\kappa=\kappa_{+}$is increased by both $k$ and $\varepsilon$. Equation (48) shows that the critical value $k_{c r}$ is decreased when $\varepsilon$ is increased, with $k_{c r}$ being negative if $\varepsilon>1$.

The input of the reaction term to $\Lambda$ is given by Eqs. (28) and (46), which result in

$$
\int_{-\infty}^{\infty} \omega(U) d \zeta= \begin{cases}2-k / 2 & \text { if } \quad-\infty<k<k_{c r} \\ (1+\varepsilon) / \kappa_{+} & \text {if } \quad k_{c r}<k<\infty\end{cases}
$$

provided that $\varepsilon \geqslant-1 / 2$. It is worth noting that at $k>k_{c r}$, the integral $\int_{-\infty}^{\infty} \omega(U) d \zeta$ can be calculated straightforwardly using Eq. (36)

$$
\begin{aligned}
\int_{-\infty}^{\infty} \omega(U) d \zeta & =\int_{0}^{1} \frac{\omega(U)}{|P|} d U=\int_{0}^{1} \frac{U(1-U)(1+2 \varepsilon U)}{\kappa_{+} U(1-U)} d U \\
& =\frac{1}{\kappa_{+}} \int_{0}^{1}(1+2 \varepsilon U) d U=\frac{1+\varepsilon}{\kappa_{+}} .
\end{aligned}
$$

In the case of $\varepsilon>1$ and $k<k_{c r}<0$, the Burgers nonlinear convection term is negative and serves to expand the profile of $U(\zeta)$. Accordingly, the integral $\int_{-\infty}^{\infty} \omega(U) d \zeta$ given by Eq. (49) in this case is increased when the magnitude $|k|$ of that term is increased. Due to the expansion of $U(\zeta)$ by negative Burgers nonlinear convection term, the profile has a long tail at the trailing edge if $k<k_{c r}<0$. Indeed, let us consider the asymptotic behavior of $U(\zeta)$ at the trailing edge, i.e., at $\zeta \rightarrow-\infty$. Substitution of

$$
\begin{aligned}
1-U & =\exp (\mu \zeta) \ll 1, \\
P & =\frac{d U}{d \zeta}=-\mu \exp (\mu \zeta)=-\kappa(1-U)<0, \\
\frac{d^{2} U}{d \zeta^{2}} & =-\mu^{2} \exp (\mu \zeta)=\mu P, \quad \mu>0
\end{aligned}
$$

into Eq. (29) yields

$$
\mu^{2}+(\Lambda-k) \mu-(1+2 \varepsilon)=0 .
$$

Therefore,

$$
\mu_{ \pm}=-\frac{\Lambda-k}{2} \pm \sqrt{\left(\frac{\Lambda-k}{2}\right)^{2}+1+2 \varepsilon}
$$

and the positive root $\mu_{+}$is decreased when the magnitude $|k|$ of negative $k$ is increased. In particular, $\mu \rightarrow 0$ when $k \rightarrow-\infty$ and the profile of $U(\zeta)$ has a long tail at the trailing edge. Similarly, the profile has a long tail at $\zeta \rightarrow-\infty$ if $\varepsilon \rightarrow-1 / 2$.

In the particular case of $\varepsilon=0$ or $k=0$, Eqs. (46) and (47) obtained in the present work reduce to equations derived earlier by Murray [3,4] or Hadeler and Rothe [37], respectively.

\section{NUMERICAL SIMULATION}

In order to support the obtained analytical results and, in particular, the steepness criterion [26-28] for selecting physically observable solutions, we numerically investigated the initial boundary value problem. Simulations were performed by using an in-house code widely exploited in previous studies, e.g., [38,39]. Briefly speaking, Eq. (16) was numerically integrated using implicit scheme for various $k$ and $\varepsilon$ on a uniform spatial grid. In order to keep the wave in the middle of the computational domain, the numerical integration was performed in the coordinate framework that moved from left to right at a speed $\lambda$ equal to $\Lambda$ given by Eq. (46). Boundary conditions were set by Eq. (17). Initial profile of $\tilde{u}(\xi, 0)$ varied linearly from 0 at $\xi=\xi_{1} \gg 1$ to unity at $\xi=\xi_{2}>\xi_{1}$, with $\xi_{2}-\xi_{1} \ll 1$. Weak sensitivity of obtained results to time step, grid size, and initial conditions was checked. The code was tested by comparing computed TW speeds with the theoretical
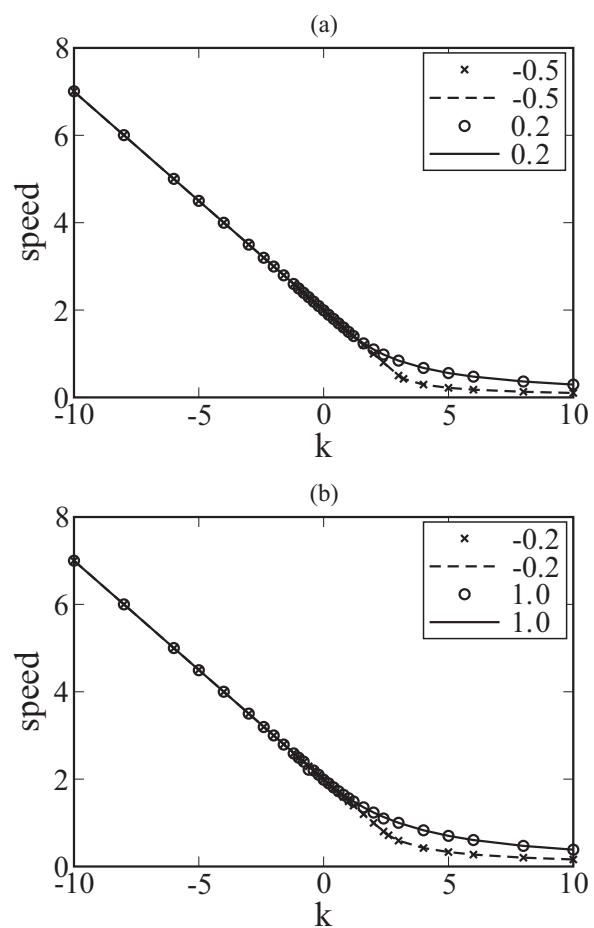

(c)

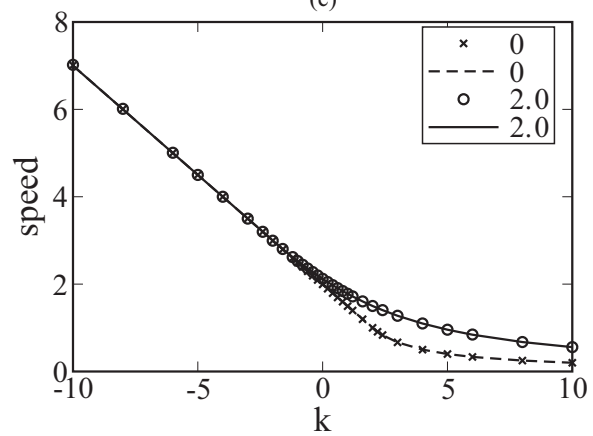

(d)

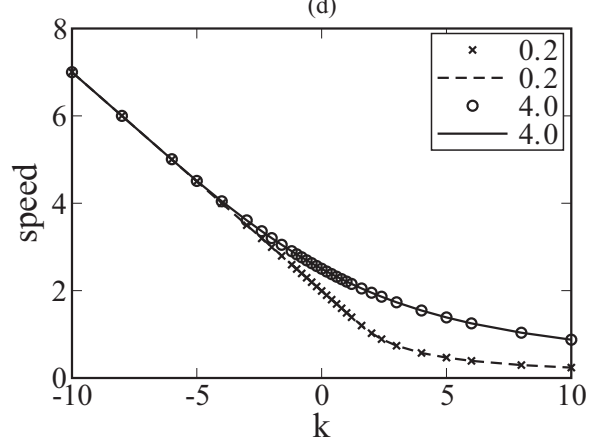

FIG. 1. TW speed vs. magnitude $k$ of the nonlinear Burgers convection term. Lines and symbols show analytical and numerical results, respectively. Parameter $\varepsilon$ is specified in legends. 
results by Kolmogorov et al. [2], analytical results by Murray [4], and numerical results by Méndez and Fort [22].

In the present paper, we restrict ourselves to discussing data computed for fully developed waves associated with TW solutions to Eq. (16) and characterized by stationary wave speed $\Lambda$ and thickness $\Delta_{t}$, which were evaluated as follows:

$$
\begin{gathered}
\Lambda=\lambda+\frac{d \xi_{f}}{d \tau}, \\
\Delta_{t}=\frac{1}{\max |d u / d \xi|},
\end{gathered}
$$

where $\xi_{f}(\tau)$ is associated with $u\left(\xi_{f}, \tau\right)=0.5$. Validity of Eq. (28) was also checked in the simulations.

Figure 1 validates the above theoretical analysis and the steepness criterion by showing that TW speeds $\Lambda$ obtained numerically in the range of $-10 \leqslant k \leqslant 10$ and $-0.5 \leqslant \varepsilon \leqslant 4$, see symbols, agree very well with the speeds $\Lambda$ given by Eq. (46), see lines. To make the validation more clear, the same results are shown solely for pushed TWs, i.e., $k \geqslant k_{c r}=$ $2(1-\varepsilon)$, in a larger scale in Fig. 2.

The exact solution given by Eq. (36) offers an opportunity to evaluate not only the speed $\Lambda$, but also the thickness $\Delta_{t}$

$$
\Delta_{t}=\frac{4}{\kappa_{+}}=\frac{16}{k+\sqrt{k^{2}+16 \varepsilon}} \quad \text { if } \quad k \geqslant k_{c r}=2(1-\varepsilon) .
$$

Figure 3 validates this analytical result and the steepness criterion invoked to arrive at Eq. (55) by showing that TW

(a)

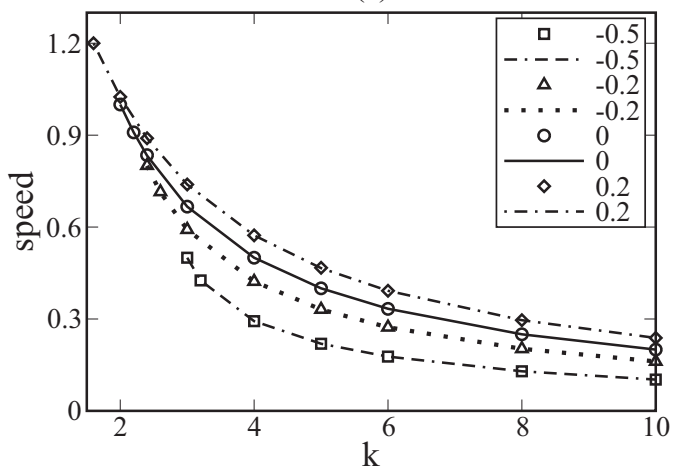

(b)

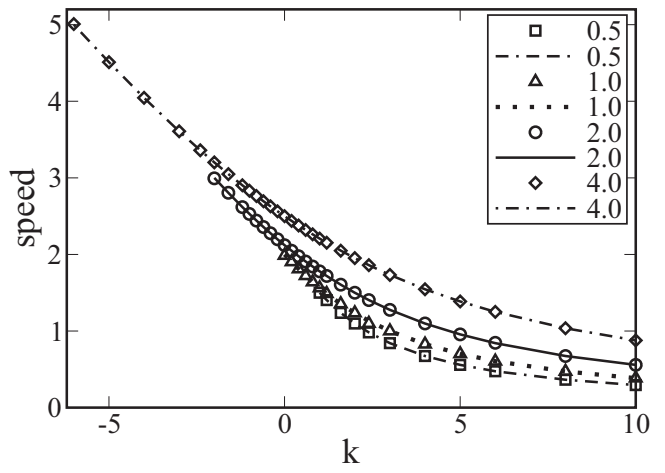

FIG. 2. Pushed TW speed vs. magnitude $k$ of the nonlinear Burgers convection term. Lines and symbols show analytical and numerical results, respectively. Parameter $\varepsilon$ is specified in legends. (a)

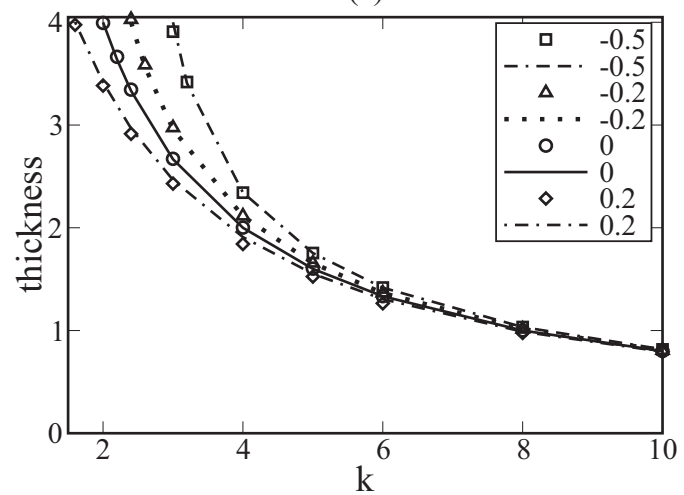

(b)

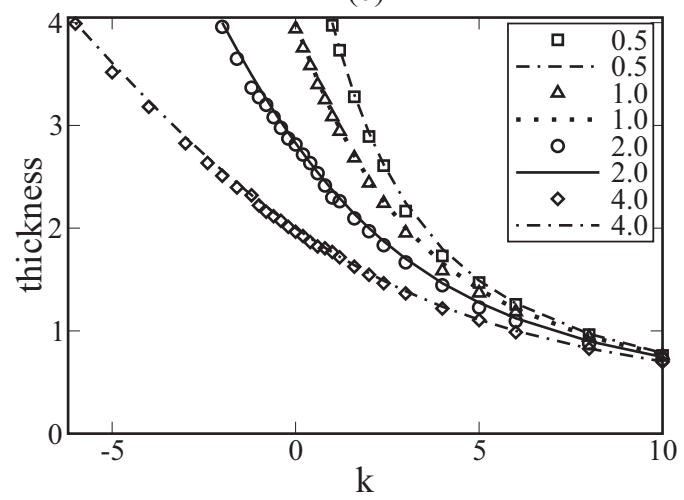

FIG. 3. Pushed TW thickness vs. magnitude $k$ of the nonlinear Burgers convection term. Lines and symbols show analytical and numerical results, respectively. Parameter $\varepsilon$ is specified in legends.

thicknesses $\Delta_{t}$ obtained numerically in the range of $k_{c r} \leqslant$ $k \leqslant 10$ and $-0.5 \leqslant \varepsilon \leqslant 4$, see symbols, agree very well with the TW thicknesses $\Delta_{t}$ given by Eq. (55), see lines.

Finally, it is worth noting that the computed structure of pushed TWs agree very well with Eq. (36) in all investigated cases such that $k \geqslant k_{c r}=2(1-\varepsilon)$. These results are not reported in a figure form, because the analytical and numerical curves are indistinguishable in all such cases. When $k<k_{c r}=$ $2(1-\varepsilon)$, the computed structure $U(\zeta)$ and thickness $\Delta_{t}$ of TWs differed from results yielded by Eqs. (36) and (55), respectively, with the differences being increased by $k_{c r}-k$. Therefore, the exact pushed TW solution discussed in Sec. IV is physically observable only if $k \geqslant k_{c r}=2(1-\varepsilon)$, in line with the contents of Sec. V.

\section{CONCLUSIONS}

By analyzing a generalized Murray-Burgers-KPP-Fisher parabolic equation with a strictly positive cubic reaction term, see Eq. (16), (i) the spectrum of traveling wave speeds was determined by linearizing the equation at the leading edge of the wave and (ii) an exact analytical solution to the equation was obtained.

The problem of selection of physically observable traveling waves was addressed by invoking a steepness criterion, i.e., by associating the observable waves with solutions characterized by the highest decay rates at their leading edges. Based on this selection criterion, the speeds of physically observable 
traveling waves were analytically determined. It was also shown that transition from pulled to pushed traveling waves occurred when the nonlinear Burgers convection term overwhelmed the nonlinear reaction term. A criterion of the transition was obtained and the thickness and structure of the pushed traveling waves were analytically found.

Numerical simulations of the counterpart initial boundary value problem fully confirmed the above theoretical results in a wide range of two problem parameters, $k$ and $\varepsilon$ in Eq. (16), thus, supporting the steepness criterion for the equation studied in the present work.

\section{ACKNOWLEDGMENTS}

The first author (V.S.) was supported by ONERA. The second author (A.L.) was supported by the Swedish Energy Agency and by the Chalmers Combustion Engine research Center (CERC).

\section{APPENDIX: APPLICATION TO PREMIXED TURBULENT COMBUSTION}

Let us consider turbulent combustion of premixed gases (fuel and air) as an example of an application of results of the present work. For simplicity, but without loss of generality, we assume that the gas density is constant. In this case, (i) Eq. (15) subsumes the well-known model developed by Bray [40] for evaluating the mean rate of product creation (note that significantly different models of the source term $\omega$ can be invoked to study other exothermic reacting wave fronts of KPP type, e.g., see $[6,7,41]$ ), while (ii) the nonlinear convection term can be associated with the so-called countergradient turbulent scalar flux [42], as discussed elsewhere [39]. Because (i) the flux should vanish for both unstable (unburned gas) and stable (products) states of the substance $u$ (the combustion progress variable $c$ ) and (ii) the flame speed $\Lambda$ should be equal to the turbulent burning velocity $\int_{-\infty}^{\infty} \omega d \zeta$, the Murray-Burgers-KPP-Fisher parabolic Eq. (16) requires a minor modification.

For this purpose, let us consider Eq. (16) in a coordinate framework that moves at a constant dimensionless speed $v$. Then, Galilean transformation $\left(\xi^{\prime}=\xi-v \tau, \tau^{\prime}=\tau\right)$ yields

$$
\frac{\partial u}{\partial \tau^{\prime}}+\frac{\partial}{\partial \xi^{\prime}}\left[\left(\frac{k}{2} u-v\right) u\right]=\frac{\partial^{2} u}{\partial \xi^{\prime 2}}+u(1-u)(1+2 \varepsilon u),
$$

because $u(\xi, \tau)$ is a scalar field. For brevity, primes in $\xi^{\prime}$ and $\tau^{\prime}$ will be skipped in the following. We can adjust the speed $v$ of the coordinate framework as follows: $v=k / 2$ in order for the nonlinear part $f(u)=(0.5 k u-v) u$ of the turbulent flux in Eq. (A1) to vanish both at $u \rightarrow 0$ or $\xi \rightarrow \infty$ and $u \rightarrow 1$ or $\xi \rightarrow-\infty$. Therefore,

$$
f=-\frac{k}{2} u(1-u)
$$

and this equation is well suited to model the nonlinear part of the scalar flux in turbulent premixed flames, provided that $k=-\operatorname{sign}(\partial u / \partial \xi)$, as discussed in detail elsewhere [39]. In order for the selected coordinate framework to be attached to the flame that moves from left to right, the parameter $k$ should be positive.

Then, Eqs. (A1), (20), (27), and (28) read

$$
\frac{\partial u}{\partial \tau}-\frac{\partial}{\partial \xi}\left[\frac{k}{2} u(1-u)\right]=\frac{\partial^{2} u}{\partial \xi^{2}}+u(1-u)(1+2 \varepsilon u),
$$

$$
\begin{gathered}
\frac{d}{d \tau} I_{u}(-\infty, \infty, \tau)=\int_{-\infty}^{\infty} \omega(u) d \xi \\
-\Lambda \frac{d U}{d \zeta}-\frac{k}{2} \frac{d U}{d \zeta}+k U \frac{d U}{d \zeta}=\frac{d^{2} U}{d \zeta^{2}}+U(1-U)(1+2 \varepsilon U)
\end{gathered}
$$

and

$$
\Lambda=\int_{-\infty}^{\infty} \omega(U) d \zeta=\int_{-\infty}^{\infty} U(1-U)(1+2 \varepsilon U) d \zeta,
$$

respectively. Equation (A6) shows that consideration of the quasi-linear convection diffusion-reaction Eq. (16) in the moving coordinate framework allows us to exclude the direct impact of the nonlinear Burgers convection term on the flame speed, which is equal to to the spatial integral of the reaction term in this framework attached to the flame.

Equations (32)-(35) read

$$
\begin{gathered}
\kappa^{2}-\left(\Lambda+\frac{k}{2}\right) \kappa+1=0, \\
\kappa_{ \pm}=\frac{1}{2}\left(\Lambda+\frac{k}{2}\right) \pm \sqrt{\frac{1}{4}\left(\Lambda+\frac{k}{2}\right)^{2}-1},
\end{gathered}
$$

and

$$
\Lambda_{\min } \geqslant \Lambda_{\mathrm{KPP}}=2-\frac{k}{2} .
$$

It is worth noting that if $k>4$, then, $\Lambda_{\mathrm{KPP}}$ given by Eq. (A9) is negative. Therefore, the linear analysis does not allow us to find the lowest eigenvalue if $k>4$.

The physically realizable TW solutions have the following speeds

$$
\Lambda= \begin{cases}2-k / 2 & \text { if } \quad 0 \leqslant k<k_{c r} ; \quad \varepsilon \geqslant-\frac{1}{2}, \\ (1+\varepsilon) / \kappa_{+} & \text {if } \quad k_{c r}<k\end{cases}
$$

while the decay rates of these solutions are still given by Eq. (47), because Galilean transformation does not change the decay rate. The critical value $k_{c r}=2(1-\varepsilon)$ is still given by Eq. (48).
[1] R. A. Fisher, Ann. Eugenics 7, 355 (1937).

[2] A. N. Kolmogorov, E. G. Petrovsky, and N. S. Piskounov, Bulleten MGU, Moscow State University, USSR, Mathematics and Mechanics, International Ser. A1 n 6, 1 (1937).
[3] J. D. Murray, Lectures on Nonlinear-Differential-Equation Models in Biology (Clarendon Press, Oxford, UK, 1977).

[4] J. D. Murray, Mathematical Biology 1. An Introduction (Springer, Berlin, 2002). 
[5] D. G. Aronson and H. F. Weinberger, in Partial Differential Equations and Related Topics, edited by J. A. Goldstein (Springer, Berlin, 1975), Lecture Notes in Mathematics, Vol. 446, p. 5.

[6] Ya. B. Zel'dovich, G. I. Barenblatt, V. B. Librovich, and G. M. Makhviladze, The Mathematical Theory of Combustion and Explosions (Plenum Publishing Corp., New York, 1985).

[7] A. G. Merzhanov and E. N. Rumanov, Rev. Mod. Phys. 71, 1173 (1999).

[8] R. J. Field and M. Burger, Oscillations and Traveling Waves in Chemical Systems (Wiley Interscience, New York, 1985).

[9] V. Méndez, S. Fedotov, and W. Horsthemke, Reaction-Transport Systems: Mesoscopic Foundations, Fronts, and Spatial Instabilities (Springer, Berlin, 2010).

[10] M. C. Cross and P. C. Hohenberg, Rev. Mod. Phys. 65, 851 (1993).

[11] A. I. Volpert, V. A. Volpert, and V. A. Volpert, Traveling Wave Solutions of Parabolic Systems (American Mathematical Society, Providence, RI, 2000).

[12] S. V. Petrovskii and B.-L. Li, Exactly Solvable Models of Biological Invasion (CRC Press, Boca Raton, FL, 2005).

[13] U. Ebert and W. van Saarloos, Physica D 146, 1 (2000).

[14] G. I. Barenblatt, Scaling, Self-Similarity, and Intermediate Asymptotics (Cambridge University Press, Cambridge, 1996).

[15] J. P. Keener and J. Sneyd, Mathematical Physiology, II. Systems Physiology (Springer, New York, 2008).

[16] J. D. Murray, J. Fluid Mech. 44, 315 (1970).

[17] T. Nagai and T. Ikeda, J. Math. Biol. 30, 169 (1991).

[18] F. S. Berezovskaya and G. P. Karev, Physics-Uspekhi 42, 917 (1999).

[19] P. D. Lax, Hyperbolic Systems of Conservation Laws and the Mathematical Theory of Shock Waves (SIAM, Philadelphia, 1973).

[20] J. Smoller, Shock Waves and ReactionDiffusion Equations (Springer-Verlag, New York, 1994).
[21] G. A. Chechkin and A. Yu. Goritsky, in Analytical and Numerical Aspects of Partial Differential Equations, edited by E. Emmrich and P. Wittbold (De Gruyter, Berlin, 2009), p. 1.

[22] V. Méndez and J. Fort, Phys. Rev. E 64, 011105 (2001).

[23] G. T. Dee and J. S. Langer, Phys. Rev. Lett. 50, 383 (1983).

[24] E. Ben-Jacob, H. R. Brand, G. Dee, L. Kramer, and J. S. Langer, Physica D 14, 348 (1985).

[25] W. van Saarloos, Phys. Rev. Lett. 58, 2571 (1987).

[26] W. van Saarloos, Phys. Rev. A 37, 211 (1988).

[27] G. T. Dee and W. van Saarloos, Phys. Rev. Lett. 60, 2641 (1988).

[28] W. van Saarloos, Phys. Rev. A 39, 6367 (1989).

[29] R. D. Benguria and M. C. Depassier, Phys. Rev. E 50, 3701 (1994).

[30] G. C. Paquette, L.-Y. Chen, N. Goldenfeld, and Y. Oono, Phys. Rev. Lett. 72, 76 (1994).

[31] A. Goriely, Phys. Rev. Lett. 75, 2047 (1995).

[32] R. D. Benguria and M. C. Depassier, Phys. Rev. Lett. 77, 1171 (1996).

[33] R. D. Benguria and M. C. Depassier, Commun. Math. Phys. 175, 221 (1996).

[34] W. van Saarloos, Phys. Rep. 386, 29 (2003).

[35] R. FitzHugh, Biophysical J. 1, 445 (1961).

[36] J. Nagumo, S. Arimoto, and S. Yoshizawa, Proc. IRE 50, 2061 (1962).

[37] K. P. Hadeler and F. Rothe, J. Math. Biology 2, 251 (1975).

[38] A. N. Lipatnikov and J. Chomiak, Combust. Theory Modell. 8, 211 (2004).

[39] V. A. Sabelnikov and A. N. Lipatnikov, Combust. Theory Modell. 17, 1154 (2013).

[40] K. N. C. Bray, Proc. R. Soc. London A 431, 315 (1990).

[41] A. Lemarchand, B. Nowakowski, G. Dumazer, and C. Antoine, J. Chem. Phys. 134, 034121 (2011).

[42] D. Veynante, A. Trouvé, K. N. C. Bray, and T. Mantel, J. Fluid Mech. 332, 263 (1997). 